ИЗВЕСТИЯ АҚАДЕМИИ НАУК ЭСТОНСКОП ССР. ФИЗИКА * МАТЕМАТИКА PROCEEDINGS OF THE ACADEMY OF SCIENCES OF THE ESTONIAN SSR.

\title{
КОГЕРЕНТНАЯ ФОТОНАМАГНИЧЕННОСТЬ В НЕЦЕНТРОСИММЕТРИЧНОМ КРИСТАЛЛЕ
}

A. PISTSEV. KOHERENTNE FOTOMAGNEETUMUS MITTETSENTRAALSOMMEETRILISES KRISTALLIS

A. PISHCHEV. COHERENT PHOTOMAGNETIZATION IN A NONCENTROSYMMETRIC CRYSTAL

\author{
(Представил В. Хижняков)
}

Взаимодействие света с электронной подсистемой нецентросимметричного кристалла обуславливает целый ряд специфических нелинейных по амплитуде возбуждающего поля явлений. Так, например, генерация второй гармоники и оптическое выпрямление достаточно широко известны. В последнее время интенсивно исследуется аномальный фотовольтаический ток [ $\left.{ }^{1}\right]$, механизм которого имеет две составляющие - баллистическую $\left[{ }^{2}\right]$ и когерентную (сдвиговую) $\left[{ }^{3-6}\right]$. Последняя не зависит от времен релаксации носителей и определяется интерференцией амплитуд реальных и виртуальных квантовых переходов, что приводит к эффективному сдвигу носителей в реальном пространстве.

В настоящем сообщении будет рассмотрен новый фотовольтаический эффект - индуцирование линейно-поляризованным светом намагниченности в немагнитном кристалле без центра симметрии. Экспериментально этот эффект наблюдался в $\left[{ }^{7}\right]$. сти $\mathrm{M}$

Исходим из общей формулы $\left[^{8}\right]$ для индуцированной намагниченно-

$$
\mathbf{M}=\sum_{\alpha, \beta}\left(\frac{\partial \varepsilon_{\alpha \beta}}{\partial \mathbf{H}}\right)_{0} \frac{\mathcal{E}_{\alpha} \varepsilon_{\beta}}{8 \pi},
$$

где $\varepsilon \alpha \beta(\omega)$ - тензор высокочастотной диэлектрической проницаемости, $\mathcal{E}_{\alpha}$ - амплитуда напряженности электрического поля световой волны, и значение производной берется при напряженности внешнего постоянного магнитного поля $\mathbf{H}=0$. В отсутствии магнитного поля при линейной поляризации световой волны в направлении $\beta \varepsilon_{\beta \beta}(\omega)$ определяется формулой

$$
\begin{aligned}
& \varepsilon_{\beta \beta}(\omega)-1=\frac{4 \pi e^{2}}{V(m \omega)^{2}} \sum_{\varepsilon= \pm 1} \sum_{\mathbf{k}} \sum_{n, l} \frac{f_{n}(\mathrm{k})-f_{l}(\mathrm{k}+\varepsilon \mathbf{q})}{E_{n}(\mathrm{k})-E_{l}(\mathrm{k}+\varepsilon \mathbf{q})+\varepsilon \omega} \times \\
& X\left|p_{n l}^{\beta}(\mathrm{k}, \mathrm{k}+\varepsilon \mathbf{q})\right|^{2},
\end{aligned}
$$

где $f_{n}-$ фермиевские числа заполнения, $V$ - объем кристалла. Случай $\mathbf{q}=0$ соответствует дипольному приближению.

Во внешнем пөстоянном магнитном поле матричные элементы переходов в (2) приобретают магнитные добавки

$$
\pi_{n l}^{\beta}(\mathrm{k}, \mathrm{k}+\varepsilon \mathrm{q})=p_{n l}^{\beta}(\mathrm{k}, \mathrm{k}+\varepsilon \mathrm{q})-\frac{e}{2 c} \frac{\partial}{\partial k_{\beta}}\left(\mathbf{L}_{n l}(\mathrm{k}, \mathrm{k}+\varepsilon \mathrm{q}) \mathbf{H}\right),
$$

где $\mathbf{L}_{n l}$ - межзонный матричный элемент оператора орбитального момента $\hat{\mathbf{L}}=\hat{\mathbf{r}} \times \hat{\mathbf{p}}$. Формула (3) получается дифференцированием по $\mathbf{k}$ недиагональных магнитоиндуцированных орбитальных вкладов в элект- 
ронный гамильтониан. Согласно (1)-(3) для средней плотности намагниченности имеем $\left(C=e^{3}\left(4 \pi^{2} m^{2} c^{2}\right)^{-1}, I=\varepsilon_{\beta}^{2} / 8 \pi\right)$

$$
M_{\alpha}=-\frac{C I m}{c \omega^{2}} \sum_{\varepsilon= \pm 1} \sum_{n, l} \int d^{3} \mathrm{k} \frac{\left[f_{n}(\mathbf{k})-f_{l}(\mathbf{k}+\varepsilon q)\right]}{E_{n}(\mathbf{k})-E_{l}(\mathbf{k}+\varepsilon \mathbf{q})+\varepsilon \omega} \times
$$

$\times\left\{p_{n l}^{\beta}(\mathrm{k}, \mathrm{k}+\varepsilon \mathbf{q}) \frac{\partial L_{l n}^{\alpha}(\mathrm{k}+\varepsilon \mathbf{q}, \mathrm{k})}{\partial k_{\beta}}+p_{l n}^{\beta}(\mathrm{k}+\varepsilon \mathbf{q}, \mathrm{k}) \frac{\partial L_{n l}(\mathrm{k}, \mathrm{k}+\varepsilon \mathbf{q})}{\partial k_{\beta}}\right\}$.

По симметрийным соображениям для центросимметричного кристалла $M_{\alpha}$ обращается тождественно в нуль вследствие свойств $p_{n l}^{\beta}(\mathrm{k}, \mathrm{k}+\varepsilon \mathbf{q})=-p_{l n}^{\beta}(\mathrm{k}+\varepsilon \mathbf{q}, \mathrm{k})$ и $L_{n l}^{\alpha}(\mathrm{k}, \mathrm{k}+\varepsilon \mathbf{q})=L_{l n}^{\alpha}(\mathbf{k}+\varepsilon \mathbf{q}, \mathbf{k}) . \quad$ В нецентросимметричном кристалле не зависящие от $\mathbf{q}$ и четные по q компоненты $M_{\alpha}$ выпадают из-за нечетности подынтегрального выражения в (4) по k. Не учтенное в (4) изменение зонных энергий, пропорциональное $\mathbf{H}$, не является существенным, поскольку при суммировании по $n$ и $l$ эти вклады выпадают.

В двухзонной аппроксимации для матричных элементов $L_{n l}^{\alpha}=$ $=\frac{1}{2} \sum_{\gamma, \sigma} \varepsilon_{\alpha \gamma \sigma} x_{n l}\left(p_{n n}^{\sigma}+p_{l l}^{\sigma}\right) \quad$ в линейном по q приближении главный вклад в формулу (4) будет давать член с $p_{l n}^{\beta} \frac{\partial p_{n l}^{\gamma}}{\partial k_{\beta}} \frac{\partial p_{l l}^{\sigma}}{\partial k_{\sigma}} q_{\sigma}$, так что в итоге $\left(\Delta_{n l}=E_{n}-E_{l}\right.$ и зависимость от $\mathbf{k}$ подынтегральных величин не выписывается)

$$
\begin{aligned}
& M_{\alpha} \simeq \frac{i C I}{2 c \omega} \sum_{n, l}^{\prime} \sum_{\gamma, \sigma} \varepsilon_{\gamma \alpha \sigma} q_{\sigma} \int d^{3} \mathrm{k} \frac{f_{n}-f_{l}}{\Delta_{n l}\left(\Delta_{n l}^{2}-\omega^{2}\right)} \times \\
& \times\left[p_{n l}^{\beta} \frac{\partial p_{l n}^{\gamma}}{\partial k_{\beta}}-p_{l n}^{\beta} \frac{\partial p_{n l}^{\gamma}}{\partial k_{\beta}}\right] \frac{\partial}{\partial k_{\sigma}}\left(p_{l l}^{\sigma}-p_{n n}^{\sigma}\right) .
\end{aligned}
$$

Когерентная намагниченность $M_{\alpha}$, изображенная формулами (4) и (5), имеет орбитальную природу и не ограничена в отличие от спиновой $\left[{ }^{7}\right]$ (рассматриваются индуцированные светом реальные переходы электронов с переворотом спина) спектральной областью межзонных переходов. Ближе к краю поглощения $M_{\alpha}$ резко возрастает и затем в области собственного поглощения постепенно уменьшается. При этом дисперсионное поведение орбитальной и спиновой намагниченностей должно быть различным.

Автор благодарен Н. Кристофелю за внимание к настоящей работе.

\section{Л И Т Е Р А Т У Р А}

1. Фридкин В. М. Фотосегнетоэлектрики. М., Наука, 1979.

2. Белиничер В. Н., Стурман Б. Н. // Успехи физ. наук, 1980, 130, вып. 3, 415-458.

3. Кристофель Н., Гулбис А. // Изв. АН ЭССР. Физ. Матем., 1979, 28, № 3, 268271.

4. Baltz, R. von, Kraut, W. von // Phys. Rev. B., 1979, 19, № 3, 1548-1554; 1981, 23, № $10,5590-5596$.

5. Kristoffel. N., Baltz, R. von, Hornung, D. // Z. Phys. B., 1982, 47, № 2, 293-296.

6. Бурсиан Э. В., Гириберг Я. Г., Трунов Н. Н. // Ж. эксперим. и теор. физ., 1982, 83, вып. 4, 1170-1175.

7. Бурсиан Э. В., Гириберг Я. Г., Егоров В. А., Каллимуллин Р. Х. // Письма в ЖЭТФ, 1983, 37, вып. 11, 520-522.

8. Ландау Л. Д., Лифииц Е. М. Электродинамика сплошных сред. М., Наука, 1982.
Ннститут физики
Академии наук Эстонской ССР
Поступила в редакцию 11/II 1988 